\title{
The appearance of pulmonary alveolar microlithiasis on dual- energy X-ray absorptiometry
}

\author{
Ahmed Fathala, Alaa Alduraibi \\ Department of Radiology, King Faisal Specialist Hospital \& Research Center, Riyadh, Saudi Arabia
}

DOI: $10.18621 /$ eurj.376484

\begin{abstract}
Dual-energy X-ray absorptiometry (DEXA) scan has been widely used as standard method of assessing bone density. Artefacts and incidental findings are frequently encountered on the DEXA scan images, some of which may affect bone mineral density values and the others are only of incidental findings. In this case report, we present a 44-year-old male diagnosed with pulmonary microlithiasis that was confirmed on a transbronchial biopsy. To our knowledge, we report the first case in the literature, describing the appearance of pulmonary alveolar microlithiasis on DEXA scan with brief review of literature on both pulmonary alveolar microlithiasis and artifacts and incidental findings encountered on DEXA scan.
\end{abstract}

Keywords: Dual-energy X-ray absorptiometry, artefacts, incidental findings, pulmonary alveolar microlithiasis

Received: January 9, 2018; Accepted: February 26, 2018; Published Online: March 30, 2018

I ncidental findings can be defined as findings that are unrelated to the clinical indications for the imaging examination. The frequency of the incidental findings depends on the imaging modalities, body regions, and age of the patients. The majority of the incidental findings in the published studies focused on computed tomography (CT) and magnetic resonance imaging (MRI). There are very few studies that reported the prevalence, clinical significance of incidental findings on dual-energy X-ray absorptiometry (DEXA). DEXA belongs to same family of imaging techniques, based as it is a conventional X-ray source of energy with similar physical and technological principles. Pulmonary alveolar microlithiasis (PAM) is a rare disease characterized by accumulation of intra-alveolar minute calculi called microliths [1]. PAM is frequently described incidentally in subjects undergoing chest radiography for other reasons. We report, a case of
PAM that was seen on DEXA scan, with brief literature review of both PAM and incidental findings of DEXA scan.

\section{CASE PRESENTATION}

A 44-year-old male diagnosed with pulmonary microlithiasis that was confirmed on a transbronchial biopsy. He was referred to our hospital for lung transplant workup. His main complaint was exertional dyspnea and breathless on walking long distance and climbing stairs. Routine work up for lung transplant included chest X-ray, chest CT, pulmonary function test, perfusion lung scan, and DEXA scan. Chest Xray revealed diffuses bilateral micronodular opacities and apical pleural calcifications consistent with pulmonary microlithiasis (Figure 1). Chest CT showed bilateral alveolar calcification in keeping with the

Address for correspondence: Ahmed Fathala, MD., King Faisal Specialist Hospital and Research Center, Medical Imaging Service/Department of Radiology, MBC\#28, 3354 Riyadh, Saudi Arabia

E-mail:ahm35799@hotmail.com,Tel:+966552532402Fax:+966144224841 


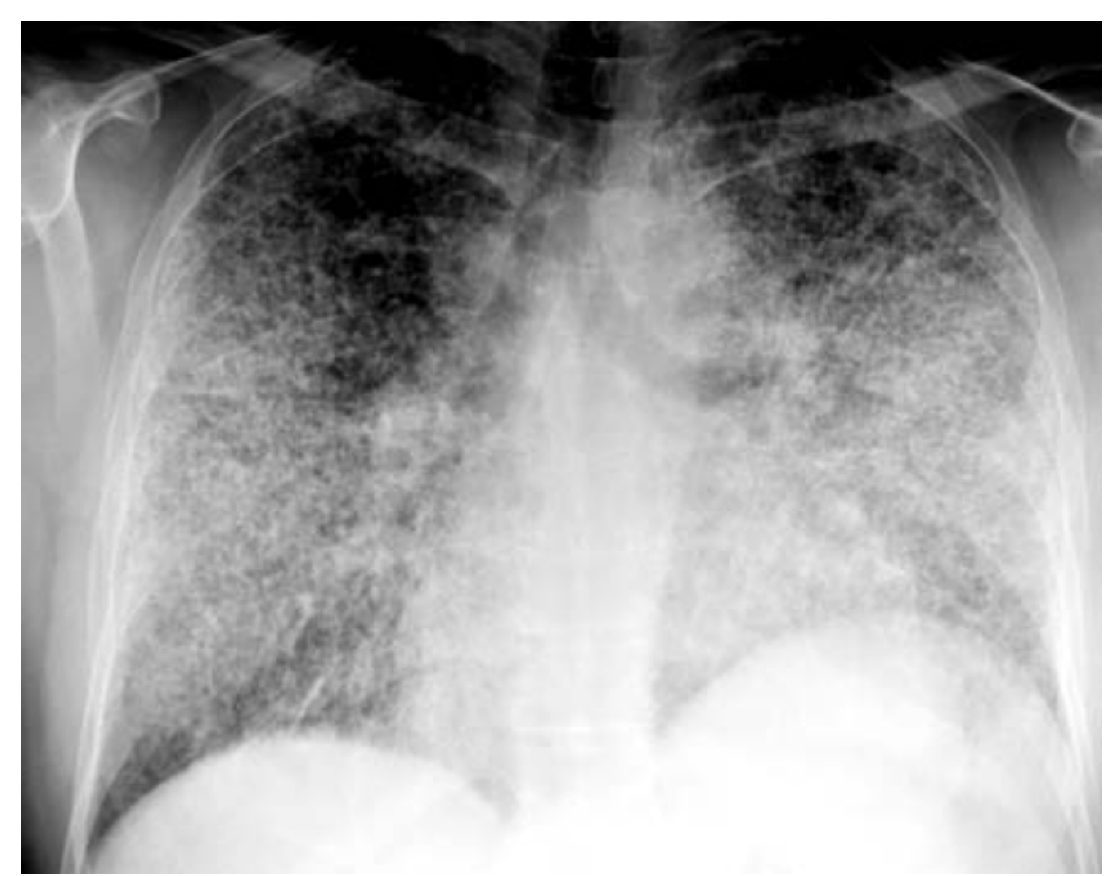

Figure 1. Posterior anterior chest X-ray of the patient showing bilateral symmetrical micronodular alveolar opacities.

diagnosis of pulmonary microlithiasis (Figure 2). Bone densitometry showed normal $\mathrm{Z}$ and $\mathrm{T}$ scores with extensive dense miliary shadow involving both lungs (Figure 3). His perfusion lungs scan showed heterogeneous lung perfusion bilaterally without evidence of pulmonary embolism (images not shown), His pulmonary function tests were consistent with restrictive lung disease.

\section{DISCUSSION}

PAM is a rare autosomal recessive that is characterized by wide spread intra-alveolar accumulation of innumerable minute calculi called microliths [1]. The disease affects both sexes, with a slight predominance among male worldwide. Although cases of PAM described at all ages, the

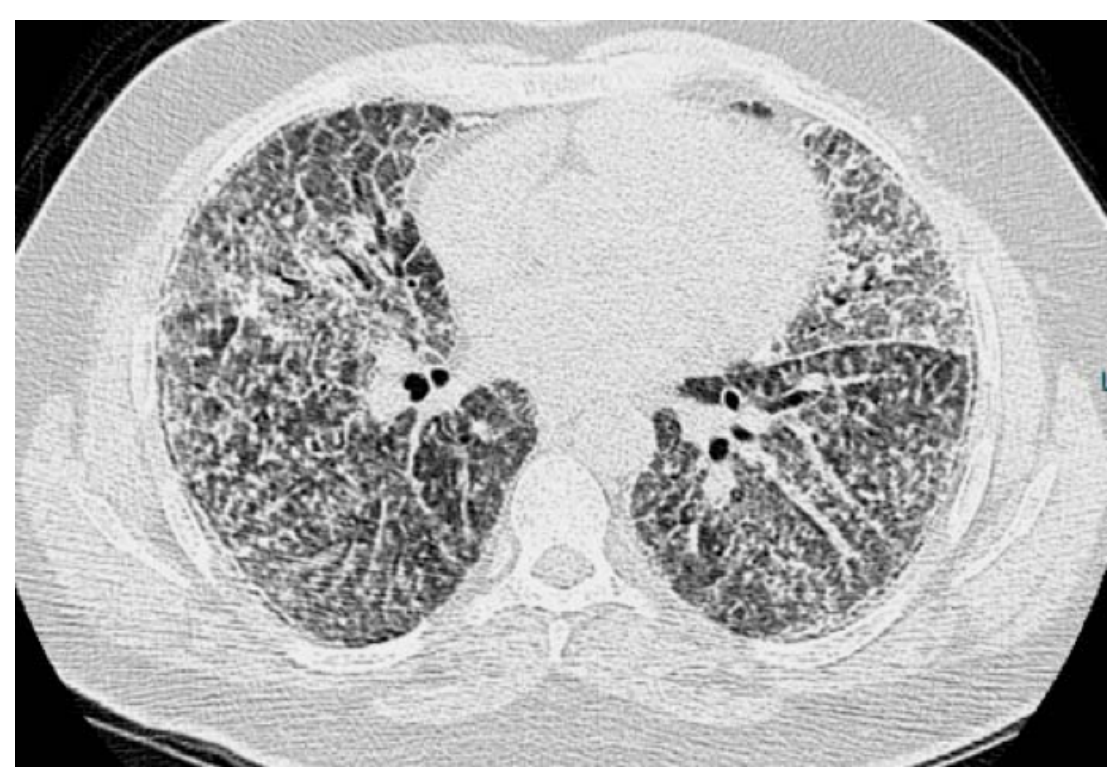

Figure 2. Lung window of the chest CT of the patient demonstrating bilateral parenchymal micronodular opacities and ground glass appearance. 


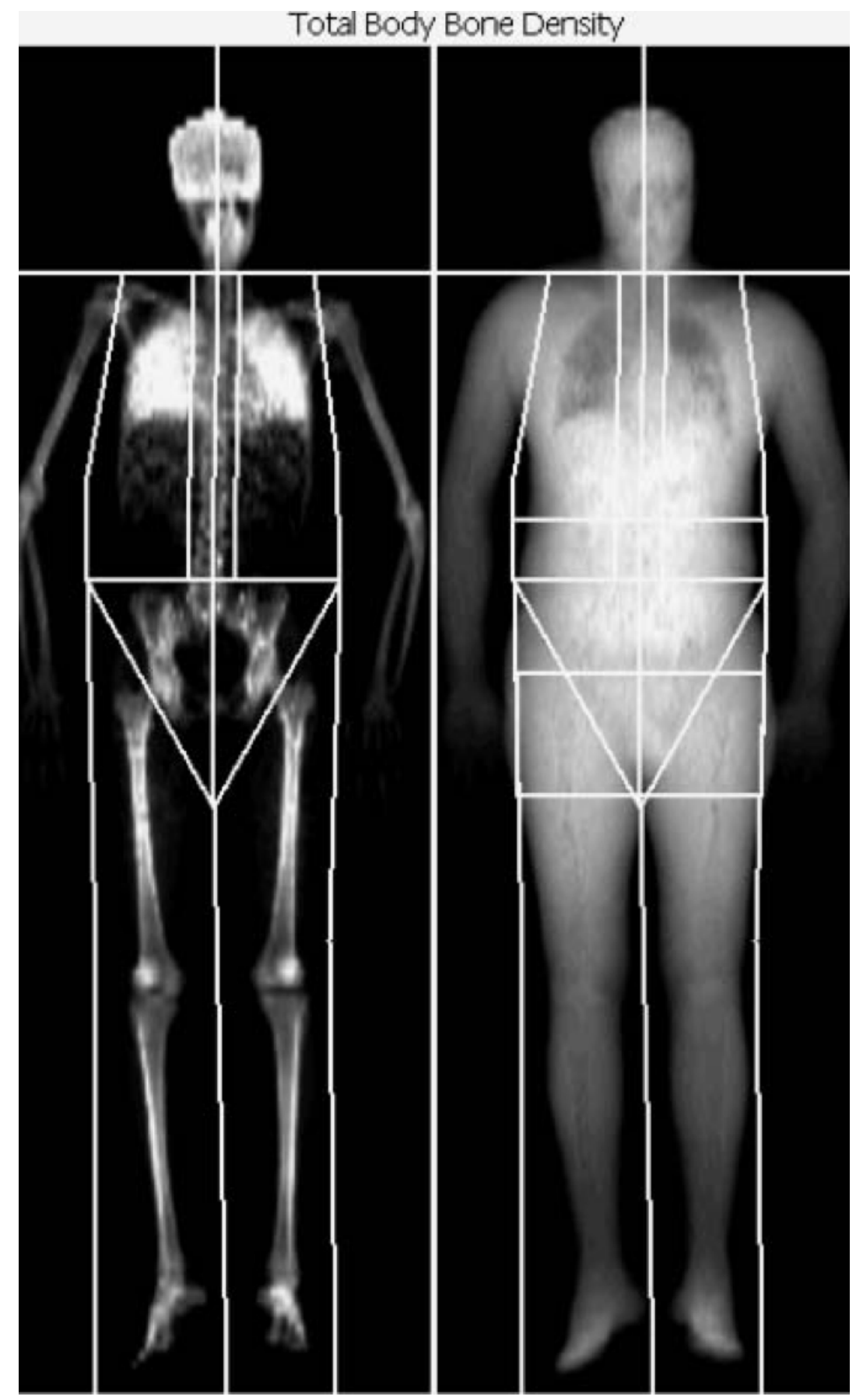

Figure 3. Whole body DEXA scan demonstrates bilateral lung parenchymal opacification consistent with known pulmonary alveolar microlithiasis.

second and third decade were found to be more frequently involved and very few cases in the elderly. Several report have a familial occurrence, with a frequency ranging between $32 \%$ to $61 \%$ [2]. The clinical presentations are very heterogeneously, ranging from totally asymptomatic to progressive respiratory failure [3]. The clinical course of the disease varies from being stationary in term of both symptoms and of the radiological and functional findings to cor pulmonale and respiratory failure. Diagnosis of PAM may be established by both radiological tests and pathological samples, such as bronchoalveloar lavage, transbronchial biopsy, and open lung biopsy.

Radiological diagnosis is usually made as an incidental finding in chest X-ray performed for 
unrelated reasons. Chest X-ray typically shows sandlike calcification distributed through the lungs, bilateral distribution with middle to lower zone predilection, and black pleural lines (Figure 1) [4]. High-resolution CT shows numerous sand-like calcification through the lungs with pleural and peribronchial distribution (typically $1 \mathrm{~mm}$ ), other additional high-resolution CT features include: crazy paving pattern, calcific interlobular septae, small subpleural cysts, black pleural signs, and ground glass opacities that tend to be more common in children (Figure 2) [5]. The bone scintigraphy scanning agents, such as technetium (Tc)-99m methylene diphosphonate shows diffuse bilateral uptake of radiotracer throughout the lung parenchyma especially in early phases of the disease when not typical chest X-ray findings are detected [6]. PAM may be seen on DEXA scan as diffuse bilateral pulmonary calcification (Figure 3). On MRI the calcific lesions usually show hypointensity or signal void on T1- and T2-weighted images. Interstitial fibrosis and thickened alveolar walls are often seen in advanced stages of PAM, but these changes show higher signal intensity on T2- than T1-weighted images [7]. Low ${ }^{18} \mathrm{~F}-$ flurodeoxyglucose (FDG) uptake on positron emission computed tomography (PET) is noted in calcification of PAM and lower FDG accumulation in the lung parenchyma without micronodular opacities. It has been postulated that higher FDG uptake may be a poor prognosis predictor for PAM [8].

In the literature studies dealing with incidental findings on DEXA mainly case reports and few single center retrospective studies. Bazzochi et al. [9], retrospectively and randomly reviewed 739 DEXA examinations. The population included 208 male and 531 female. Incidental findings were detected in 117 (15.8\%) of 739 examinations. In all cases the key markers used to detect new information from DEXA examination we represented by change in density or shape of tissue. In 76 (65\%) of all detected findings, calcifications were the primary sign of physiological or pathological process, in $41(35 \%)$ anatomical features and morphology guided identification. However, only 50 among all incidental findings could be visualized by other imaging modalities [9]. There are several non-osseous causes of elevated bone mineral density measurements. For example, the effects of aortic calcifications on lumbar spine bone mineral density is much discussed the literature, though not universal, most studies reported that vascular calcification have no significant effects on bone mineral density [10]. Cholelithiasis, nephrolithiasis, and calcific leiomyofibroma are frequently seen on DEXA such as porcelain gall bladder, mesenteric calcifications, phleoboliths, and calcinosis cutis [11]. Heterotopic calcification of the hip has been described as causes of apparent increase in bone mineral density [12]. These extra-skeletal calcifications represent a common manifestation of several pathological processes.

\section{CONCLUSION}

In summary, PAM is a rare genetic disease characterized by numerous intra-alveolar accumulations of microliths. Diagnosis of PAM can be made on the basis of the typical chest X-ray and chest CT. However, it may be seen as incidental finding in many other imaging modalities such as bone scan and DEXA scan.

\section{Informed consent}

Written informed consent was obtained from the patient for the publication of this case report.

\section{Conflict of interest}

The authors declared that there are no potential conflicts of interest with respect to the research, authorship, and/or publication of this article.

\section{REFERENCES}

[1] Barbolini G, Rossi G, Bisetti A. Pulmonary alveolar microlithiasis. N Eng J Med 2002;347:69-70.

[2] Sosman MC, Dodd GD, Jones WD, Pillmore GU. The familial occurrence of pulmonary alveolar microlithiasis. Am J Roentgenol Radium Ther Nucl Med 1957;77:947-1012.

[3] Mariotta S, Ricci A, Papale M, De Clementi F, Sposato B, Guidi L, et al. Pulmonary alveolar microlithiasis: report on 576 cases published in the literature. Sarcoidosis Vasc Diffuse Lung Dis 2004;21:173-81.

[4] Helbich TH, Wojnarovsky C, Wunderbaldinger P, Heinz-Peer G, Eichler I, Herold CJ. Pulmonary alveolar microlithiasis in children: radiographic and high-resolution CT findings. AJR Am J Roentgenol 1997; 168:63-5.

[5] Gasparetto EL, Tazoniero P, Escuissato DL, Marchiori E, Frare E Silva RL, Sakamoto D. Pulmonary alveolar microlithiasis presenting with crazy-paving pattern on high resolution CT. Br J Radiol 
2004;77:974-6.

[6] Fallahi B, Ghafary B, Fard-Esfahani A, Eftekhari M. Diffuse pulmonary uptake of bone-seeking radiotracer in bone scintigraphy of a rare case of pulmonary alveolar microlithiasis. Indian J Nucl Med 2015;30:277-9.

[7] Hoshino H, Koba H, Inomata S, Kurokawa K, Morita Y, Yoshida K, et al. Pulmonary alveolar microlithiasis: high-resolution CT and MR findings. J Comput Assist Tomogr 1998;22:245-8.

[8] Ito K, Kubota K, Yukihiro M, Izumi S, Miyano S, Kudo K, et al. FDG-PET/CT finding of high uptake in pulmonary alveolar microlithiasis. Ann Nucl Med 2007;21:415-8.

[9] Bazzocchi A, Ferrari F, Diano D, Albisinni U, Battista G, Rossi C, et al. Incidental findings with dual-energy X-ray absorptiometry: spectrum of possible diagnoses. Calcif Tissue Int 2012;91:149-56.

[10] Frye MA, Melton LJ 3rd, Bryant SC, Fitzpatrick LA, Wahner HW, Schwartz RS, et al. Osteoporosis and calcification of the aorta. Bone Miner 1992;19:185-94.

[11] Martineau P, Bazarjani S, Zuckier LS. Artifacts and incidental findings encountered on dual-energy X-ray absorptiometry: atlas and analysis. Semin Nucl Med 2015;45:458-69.

[12] Stewart CA, Hung GL, Garland DE, Rosen CD, Adkins R. Heterotopic ossification. Effect on dual-photon absorptiometry of the hip. Clin Nucl Med 1990;15:697-700. 